\title{
Coronary Computed Tomography Angiography-Based Coronary Risk Stratification in Subjects Presenting With No or Atypical Symptoms
}

\author{
Shinichiro Fujimoto, MD, PhD; Takeshi Kondo, MD, PhD; Takahide Kodama, MD, PhD; \\ Tadaaki Orihara, MD; Junichi Sugiyama, MD; Makoto Kondo, MD; Akira Endo, MD, PhD; \\ Hiroshi Fukazawa, MD; Hideki Nagaoka, MD, PhD; Akitsugu Oida, MD, PhD; \\ Takanori Ikeda, MD, PhD; Junichi Yamazaki, MD, PhD; \\ Shinichi Takase, MD; Jagat Narula, MD, PhD
}

\begin{abstract}
Background: Coronary computed tomography angiography (CTA) findings of positive vessel remodeling and lowattenuation plaque, referred to as computed tomography-verified high-risk plaque (CT-HRP), have been reported to be associated with the development of subsequent acute coronary syndromes. The aim of this study was to examine the usefulness of coronary CTA for coronary risk re-stratification of patients with asymptomatic and atypical chest symptoms.
\end{abstract}

Methods and Results: A total of 1,139 subjects (M/F 602/537; mean age, 61.5 \pm 9.3 years) who were either asymptomatic or presented with atypical chest symptoms underwent coronary 64- or 320-slice multidetector computed tomography angiography and Agatston score. Age, sex, coronary risk factors, including hypertension, diabetes mellitus (DM), dyslipidemia, and smoking were investigated as predictors for CT-HRP on multivariate analysis using logistic regression analysis. CT-HRP was observed in 72 patients (6.3\%). Based on Framingham risk scores (FRS), CT-HRP was observed in 0/94 subjects (0.0\%) in the low-risk group, 35/806 (4.3\%) in the intermediate-risk group, and 37/239 (15.5\%) in the high-risk group. On logistic regression analysis significant predictors for CT-HRP in intermediate- and high-risk subjects were male sex (odds ratio [OR] 2.829; 95\% confidence interval [Cl] 1.460-5.479, P=0.0021), DM (OR 2.418; 95\% Cl 1.420-4.116, P=0.0011), and current smoking (OR 1.922; 95\% Cl 1.096-3.371, P=0.0160). CT-HRP prevalence for Agatston scores $>500$ and $>250$ was lower in the intermediate- and high-risk groups, respectively.

Conclusions: In asymptomatic subjects and those presenting with atypical chest pain who have a more than an intermediate risk, coronary CTA is contributory to FRS. Male sex, DM and smoking were independent predictors of vulnerable plaque in the more than intermediate-risk group. (Circ $J$ 2012; 76: 2419-2425)

Key Words: Coronary computed tomography angiography; Framingham risk score; Low-attenuation plaque; Positive remodeling; Vulnerable plaque

A cute coronary syndrome (ACS) may occur without precursor symptoms, and its prediction remains an important issue for cardiologists. Indeed, a meta-analysis of coronary angiography during a 5-year period before the onset of ACS reported that $68 \%$ of culprit lesions showed no significant stenosis. ${ }^{1}$ At present, there is no established method of ACS prediction. In the risk stratification of ACS, Braunwald noted that the traditional Framingham risk score (FRS) was in- sufficient, and expected the advent of a non-invasive diagnostic imaging tool that facilitates the detection of the higher-risk group with $>15 \%$ risk of developing ACS per year. ${ }^{2}$

Naghavi et al reported various types of vulnerable plaque. ${ }^{3}$ Among them, rupture-prone, vulnerable plaque, which accounts for approximately $60 \%$ of all vulnerable plaques and causes plaque rupture, is the most commonly recognized morphology of vulnerable plaque. It has been reported that, in general, vul-

Received February 9, 2012; revised manuscript received May 7, 2012; accepted June 4, 2012; released online August 1, 2012 Time for primary review: 42 days

Department of Cardiology, Takase Clinic, Takasaki (S.F., T. Kondo, T. Kodama, T.O., J.S., M.K., A.E., H.F., H.N., A.O., S.T.); Department of Cardiovascular Medicine, Toho University Omori Medical Center, Tokyo (S.F., T.I., J.Y.), Japan; and Zena and Michael A. Wiener Cardiovascular Institute and Marie-Josee and Henry R. Kravis Cardiovascular Health Center, Mount Sinai School of Medicine, New York, NY (J.N.), USA

Mailing address: Shinichiro Fujimoto, MD, PhD, Department of Cardiology, Takase Clinic, 885-2 Minami-orui, Takasaki 370-0036, Japan. E-mail: s-fujimo@tj8.so-net.ne.jp

ISSN-1346-9843 doi:10.1253/circj.CJ-12-0157

All rights are reserved to the Japanese Circulation Society. For permissions, please e-mail: cj@j-circ.or.jp 
nerable plaque is characterized by positive vascular remodeling, plaque area $>50 \%$ of the vessel's cross-sectional area, a necrotic core occupying $>25 \%$ of the plaque, vasa vasorum within the plaque, a fibrous cap $<65 \mu \mathrm{m}$ in thickness, and infiltration of the fibrous cap by macrophages expressing matrix metalloproteinases. ${ }^{4}$

In contrast, Motoyama et al reported that, compared with the culprit lesions of stable angina pectoris, those of ACS were significantly more frequently characterized by spotty calcification, positive remodeling, and the presence of low-attenuation plaque, ${ }^{5}$ and that 2 -feature positive plaque (2FPP) with positive remodeling (remodeling index $>1.1$ ) and low-attenuation plaque (computed tomography [CT] number $<30$ Hounsfield units [HU]) resulted in ACS within 2 years with a probability of $22.2 \% .^{6}$ Thus, at present, 2 FPP is most commonly recognized as rupture-prone vulnerable plaque on coronary CT angiography (CTA). Such angiographic observations, coupled with recent technological advances in CT technology to reduce the radiation exposure, ${ }^{7-11}$ may allow extension of the indications for coronary CTA for risk stratification of patients with asymptomatic and atypical chest symptoms, which we examined in the present study.

\section{Methods}

\section{Subjects}

Subjects were 1,139 consecutive patients with suspected obstructive coronary artery disease (M/F, 602/537; age range, 30-74 years; mean, 61.5 \pm 9.3 years) who underwent coronary 64- or 320-slice coronary CTA and coronary calcium scan despite being asymptomatic or having atypical chest symptoms. The subjects consisted of patients either who were suspecied angina or who had coronary CTA requested by physicians at outpatient care in the presence or absence of evidence of ischemic heart disease (eg, atypical chest symptoms, 1 or more risk factors, ECG abnormalities, preoperative evaluation or request of coronary CTA from another institution).

Patients with known coronary artery disease and those in whom the imaging was of poor quality were excluded from the study.

All subjects were classified using the $\mathrm{FRS}^{12}$ and based on that they were categorized as low $(<5 \%)$, intermediate $(5-20 \%)$, or high risk $(<20 \%)$. Age, sex, coronary risk factors, including hypertension (HT), diabetes mellitus (DM), dyslipidemia (DL), current smoking, body mass index (BMI) and lipid profile (total cholesterol, triglycerides [TG], low-density lipoprotein cholesterol [LDL-C], and high-density lipoprotein cholesterol [HDLC]) were investigated as predictors for CT-verified high-risk plaque (CT-HRP) on univariate analysis using logistic regression analysis. Age, sex, and coronary risk factors including HT, DM, DL, current smoking, TG and HDL-C, were investigated as predictors for CT-HRP on multivariate analysis using logistic regression analysis.

This study was approved by the ethics committee of Takase Clinic.

\section{Chest Symptoms}

Chest symptoms were classified as typical, atypical or none. Typical symptoms were defined $\geq 3$ of 5 characteristics based on the Duke Clinical Score. ${ }^{13,14}$ Atypical was defined as 1-2 of the 5 characteristics. None was defined as symptoms that did not include any of the 5 characteristics. The 5 characteristics were (1) precipitation by exercise or emotional stress; (2) brief duration (2-15 $\mathrm{min}$ ); (3) prompt relief with rest or nitroglycerin; (4) retrosternal location; and (5) radiating to jaw, neck or left arm.

\section{Definition of Risk Factors}

HT was defined as systemic blood pressure $\geq 140 / 90 \mathrm{mmHg}$ or the use of antihypertensive treatment. DM was defined as fasting blood sugar $\geq 126 \mathrm{mg} / \mathrm{dl}$, postprandial blood sugar $\geq 200 \mathrm{mg} / \mathrm{dl}$, hemoglobin $\mathrm{A}_{1 \mathrm{c}} \geq 6.5 \%$ or the use of treatment. DL was defined as total cholesterol $\geq 220 \mathrm{mg} / \mathrm{dl}$, LDL-C $\geq 140 \mathrm{mg} / \mathrm{dl}$, fasting TG $\geq 150 \mathrm{mg} / \mathrm{dl}$, HDL-C $<40 \mathrm{mg} / \mathrm{dl}$ or the use of lipidlowering treatment. Current smoking was defined as smoking within 1 year of coronary CTA imaging.

\section{4-Slice Multidetector Computed Tomography (MDCT) Protocol}

Subjects with heart rate $>60$ beats/min and without contraindications underwent $\beta$-blockade with $25 \mathrm{mg}$ atenolol by mouth the evening before the examination. Alternatively, heart rate control with a target of 60 beats/min was achieved using 2$10 \mathrm{mg}$ i.v. propranolol injected before data acquisition.

The tube voltage for imaging (Aquilion 64, Toshiba Medical Systems, Tochigi, Japan) was $120 \mathrm{kV}$. The tube current range was $400-600 \mathrm{~mA}$; patients with a BMI of 22 were imaged at $440 \mathrm{~mA}$, and for each 2-point increase or decrease in BMI, the tube current was increased or decreased by $10 \mathrm{~mA}$. For example, a patient with $\mathrm{BMI}=18$ was imaged at $420 \mathrm{~mA}$. The gantry rotation time $(0.35-0.45 \mathrm{~s})$ and beam pitch $(0.125-$ 0.26 ) were determined by a manufacturer-based algorithm to optimize image quality. Imaging was performed from caudal to cranial.

Between 60 and $80 \mathrm{ml}$ of iodinated contrast (300-370 mg iodine/ml; Iopamiron-370, Bayer, Osaka, Japan; Omnipaque 300 and 350, Daiichi-Sankyo, Tokyo, Japan), was injected (Stellant Dual Flow, Nihon Medrad Osaka, Japan) via an antecubital vein using a 3-phase injection method: contrast alone (12s), followed by an equal volume admixture of contrast and saline $(9 \mathrm{~s})$, and then saline only $(2.5 \mathrm{~s})$. The iodine load was weight based, and timed using manual triggering when contrast arrived in the left ventricle.

The ECG gating acquisition strategy was divided into 4 levels depending on the patient's heart rate, with the least exposure for those with the lowest heart rate $(<60$ beats/min), and progressively increasing for patients with faster heart rates.

\section{0-Slice MDCT Protocol}

Subjects with a heart rate $>60$ beats/min and without contraindications underwent $\beta$-blockade with $25 \mathrm{mg}$ atenolol by mouth the evening before the examination. Alternatively, heart rate control with a target of 60 beats/min was achieved using 2$10 \mathrm{mg}$ i.v. propranolol injected before data acquisition.

The imaging protocol for the second cohort paralleled that of the first, with the following differences: a $320 \times 0.5 \mathrm{~mm}$ scanner (Aquilion ONE, Toshiba Medical Systems) operated on a v4.51 software platform; and the craniocaudual field of view was tailored to the smallest exposure $(10 \mathrm{~cm}, 12 \mathrm{~cm}, 12.8 \mathrm{~cm}$, or $16 \mathrm{~cm}$ ) that encompassed the heart.

The default tube voltage was $120 \mathrm{kV}$. The tube current was modulated according to the patient's body habitus. Axial imaging had a gantry rotation time of $0.35-0.4 \mathrm{~s}$ per rotation.

Contrast was injected via a 2-phase protocol: contrast medium alone for $10 \mathrm{~s}$, followed by saline for $8 \mathrm{~s}$. The contrast and saline injection rates were calculated as the individual patient's mass in kilograms multiplied by $0.06 \mathrm{ml} / \mathrm{s}$.

Based on prior experience with 64-detector row coronary CTA, the ECG gating acquisition strategy was divided into 5 heart rate groups. 


\begin{tabular}{|lc|}
\hline \multicolumn{2}{|c|}{ Table 1. Baseline Clinical Characteristics of the Study } \\
Patients \\
Age (years) & $61.5 \pm 9.3$ \\
Sex (M/F) & $602 / 537$ \\
Hypertension (\%) & 60.4 \\
Dyslipidemia (\%) & 78.8 \\
Diabetes mellitus (\%) & 19.6 \\
Current smoking (\%) & 22.6 \\
Body mass index & $24.0 \pm 3.6$ \\
Agatston score & $113.1 \pm 360.0$ \\
Framingham risk score & $94 / 806 / 239$ \\
(low/intermediate/high) & \\
Total cholesterol (mg/dl) & $206.1 \pm 36.3$ \\
Triglycerides (mg/dl) & $149.6 \pm 111.2$ \\
LDL-cholesterol (mg/dl) & $126.2 \pm 33.1$ \\
HDL-cholesterol (mg/dl) & $54.4 \pm 14.1$ \\
Statins (\%) & 47.2 \\
\hline
\end{tabular}

HDL, high-density lipoprotein; LDL, low-density lipoprotein.

\section{Image Reconstruction}

Half-image reconstruction or segmental image reconstruction was performed in the slow filling phase and end-systolic phase using the $\mathrm{R}+$ absolute time method to generate images, and images with the lowest level of motion artifacts were selected on the 4-chamber cardiac cine CT.

\section{Coronary CTA Interpretation}

For plaque detection, both cross-sectional and longitudinal curved multi-planar reconstruction images were analyzed. Coronary arteries were divided into 17 segments based on the modified recommendations of the American Heart Association. ${ }^{15}$ Coronary artery segments with a diameter $>2 \mathrm{~mm}$ were evaluated for the presence of plaque. All plaque was assessed for the presence of vessel remodeling (positive, negative, none), plaque consistency (low or intermediate attenuation) and disposition of coronary calcification (spotty, moderate, severe). Three observers were involved in the interpretation of data.

\section{Definition of CT Plaque Characteristics}

Vessel Remodeling Coronary arterial remodeling was defined as a change in the vessel diameter at the plaque site in comparison to the reference segment set proximal to the lesion in a normal-appearing vessel segment (reference diameter). Manual inspection, in both the cross-section and longitudinal reconstructions, was used to define the remodeling index (lesion diameter/reference diameter). The remodeling index was reported as positive remodeling when the diameter at the plaque site was at least $10 \%$ larger than that of the reference segment.

Plaque Consistency If non-calcified plaque or plaque with an area of spotty calcification, either or which could be measured as the plaque's consistency on the axial image, the CT number, defined as the lowest CT number of 5 areas of minimum region of interest $(0.36 \times 0.36 \mathrm{~mm})$, was measured using the axial image. Low-attenuation plaque was defined as CT $<30 \mathrm{HU}$.

CT-HRP Such a plaque was defined as having positive remodeling of at least $110 \%$ and attenuation $<30 \mathrm{HU}$.

\section{Coronary Calcium Scan (Agatston Score)}

All data were evaluated using workstation software (Zio M900 or ZioStation, Ziosoft). A calcified lesion was defined as $\geq 3$

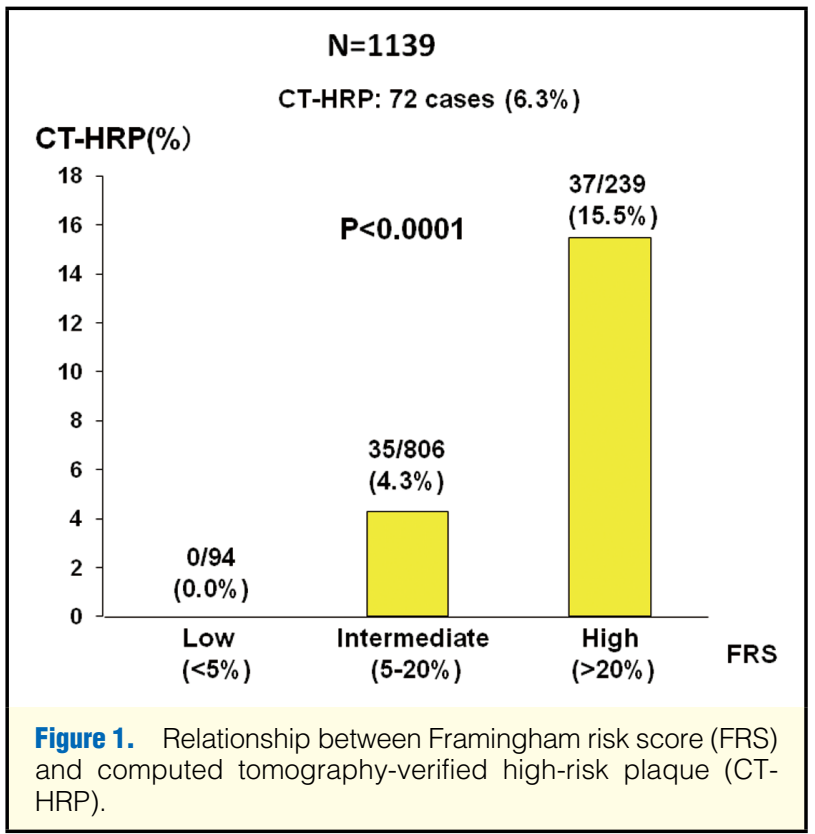

contiguous pixels with a peak attenuation $\geq 130 \mathrm{HU}$. The total coronary artery calcium score (Agatston score) was calculated as per the recommendations of Agatston et al. ${ }^{16}$

Patients were divided into 4 groups according to their Agatston scores $(0,1-250,251-500,>500)$, based on a previous report. ${ }^{17}$

\section{Statistical Analysis}

Statistical analysis was performed using Statview J-5.0 for Windows (HULINKS, Tokyo Japan). Data are expressed as mean \pm SD. To determine the statistical significance of differences between groups, the chi-square test was used for comparisons between groups. Multivariate logistic regression analysis was used to determine predictors for CT-HRP. P $<0.05$ was considered statistically significant in all instances.

\section{Results}

Baseline clinical characteristics of all subjects are listed in Table 1.

\section{FRS and CT-HRP}

CT-HRP were observed in $72(6.3 \%)$ of the 1,139 subjects. Based on FRS assignment, CT-HRP was observed in 0/94 subjects $(0.0 \%)$ in the low-risk group (FRS <5\%), 35/806 $(4.3 \%)$ in the intermediate-risk group (FRS 5-20\%), and $37 / 239(15.5 \%)$ in the high-risk group (FRS $>20 \%$; $\mathrm{P}<0.0001$; Figure 1).

\section{FRS, Agatston Score and CT-HRP}

In the intermediate- and high-risk subjects $(n=1,045), C T-H R P$ was observed according to Agatston score categories as follows: 14/430 (3.3\%), intermediate + Agatston score 0; 15/304 (4.9\%), intermediate risk + Agatston score 1-250; 4/41 (9.8\%), intermediate + Agatston score 251-500; 2/31 (6.5\%), intermediate + Agatston score $>500 ; 4 / 57(7.0 \%)$, high + Agatston score 0; 23/115 (20.0\%), high+Agatston score 1-250; 6/35 (17.1\%), high + Agatston score 251-500; and 4/32 (12.5\%), high + Agatston score $>500$ (Figure 2). The prevalence of CT-HRP in the high-risk group was significantly higher than in the inter- 


\section{$\mathrm{N}=1045$}

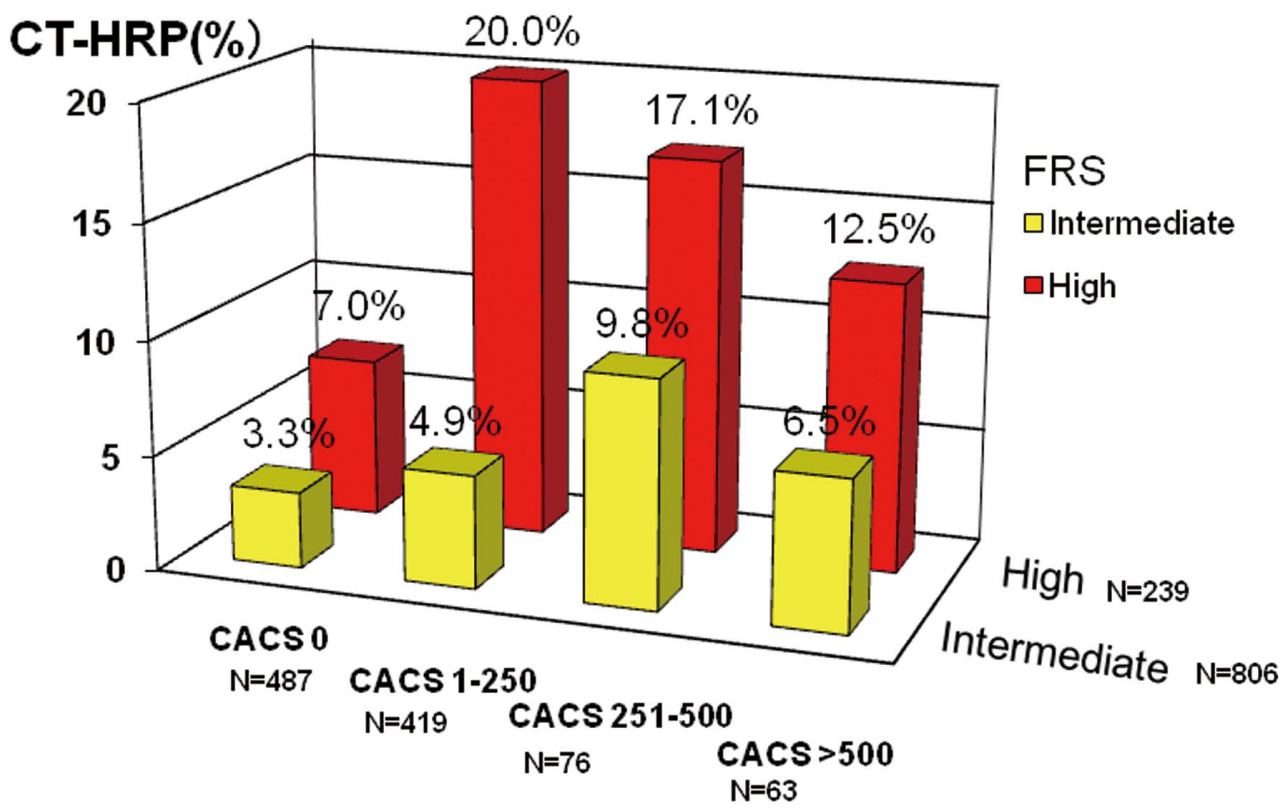

Figure 2. Relationships among Framingham risk score (FRS), Agatston score and computed tomography-verified high-risk plaque (CT-HRP).

\begin{tabular}{|lccc|}
\hline \multicolumn{5}{|c|}{ Table 2. Predictors of CT-HRP for All Subjects in the More } \\
Than Intermediate-Risk Group & \\
Univariate analysis & OR & $95 \%$ CI & P value \\
Age & & & \\
Male sex & 0.992 & $0.965-1.019$ & 0.5507 \\
Hypertension & 3.761 & $2.070-6.833$ & $<0.0001$ \\
Dyslipidemia & 1.075 & $0.650-1.777$ & 0.7774 \\
Diabetes & 1.372 & $0.708-2.658$ & 0.3483 \\
Current smoking & 2.887 & $1.761-4.733$ & 0.0001 \\
Body mass index & 2.619 & $1.604-4.276$ & 0.0001 \\
Total cholesterol & 1.055 & $0.992-1.122$ & 0.0898 \\
Triglycerides & 0.995 & $0.988-1.002$ & 0.1604 \\
LDL-cholesterol & 1.002 & $1.001-1.004$ & 0.0026 \\
HDL-cholesterol & 0.999 & $0.991-1.007$ & 0.7958 \\
Multivariate analysis & 0.979 & $0.960-0.999$ & 0.0362 \\
Age & & & \\
Male sex & 1.017 & $0.987-1.049$ & 0.2719 \\
Hypertension & 2.829 & $1.460-5.479$ & 0.0021 \\
Dyslipidemia & 1.011 & $0.587-1.739$ & 0.9695 \\
Diabetes mellitus & 1.070 & $0.525-2.183$ & 0.8520 \\
Current smoking & 2.418 & $1.420-4.116$ & 0.0011 \\
Triglycerides & 1.922 & $1.096-3.371$ & 0.0226 \\
HDL-cholesterol & 1.002 & $1.000-1.003$ & 0.0757 \\
\hline Confing & 1.004 & $0.983-1.026$ & 0.6978 \\
\hline
\end{tabular}

$\mathrm{Cl}$, confidence interval; CT-HRP, computed tomography-verified high-risk plaque; OR, odds ratio. Other abbreviations as in Table 1. mediate-risk group for Agatston score 1-250 only $(\mathrm{P}<0.0001)$.

\section{Predictors of CT-HRP}

In the intermediate- and high-risk subjects $(n=1,045)$, univariate logistic regression analysis indicated that significant predictors for CT-HRP were male sex (odds ratio [OR] 3.761; 95\% confidence interval $[\mathrm{CI}] 2.070-6.833, \mathrm{P}<0.0001), \mathrm{DM}$ (OR 2.887; 95\% CI 1.761-4.733, $\mathrm{P}<0.0001$ ), current smoking (OR 2.619; 95\% CI 1.604-4.276, $\mathrm{P}=0.0001$ ), TG (OR 1.002; 95\% CI 1.001-1.004, $\mathrm{P}=0.0026$ ), and HDL-C (OR 0.979; 95\% CI 0.960-0.999, $\mathrm{P}=0.0362$; Table 2).

On multivariate logistic regression analysis, the significant predictors for CT-HRP were male sex (OR 2.829; 95\% CI $1.460-5.479, \mathrm{P}=0.0021)$, DM (OR 2.418; 95\% CI 1.420$4.116, \mathrm{P}=0.0011$ ), and current smoking (OR 1.922; 95\% CI 1.096-3.371, $\mathrm{P}=0.0226$; Table 2). When we analyzed the data according to sex, logistic regression analysis indicated significant predictors for CT-HRP to be only current smoking (OR 1.893; 95\% CI 1.063-3.369, $\mathrm{P}=0.0302$ ) for male subjects (Table 3) and only DM (OR 9.234; 95\% CI 2.902-29.382, $\mathrm{P}=0.0002$ ) for female subjects (Table 4).

\section{Frequency of CT-HRP and Age Decades}

In the intermediate- and high-risk subjects $(\mathrm{n}=1,045), \mathrm{CT}-\mathrm{HRP}$ was found in 1/14 (7.1\%), 7/74 (9.5\%), 11/134 (8.2\%), 27/244 $(11.1 \%), 12 / 102(11.8 \%)$ of men in their $30 \mathrm{~s}, 40 \mathrm{~s}, 50 \mathrm{~s}, 60 \mathrm{~s}$, and $70 \mathrm{~s}$ or older, respectively, and in $0 / 0(0.0 \%), 0 / 5(0.0 \%)$, $3 / 93(3.2 \%), 8 / 247(3.2 \%), 3 / 132(2.3 \%)$ of women in the respective decades. In men, the frequency of CT-HRP was approximately $10 \%$ in all age groups, but in the women, it was approximately $3 \%$ in those in their $50 \mathrm{~s}$ and older. CT-HRP 


$\begin{aligned} & \text { Table 3. Multivariate Analysis for Predictors of CT-HRP in } \\
& \text { Male Patients in the More Than Intermediate-Risk } \\
& \text { Group }\end{aligned}$
\begin{tabular}{lccc} 
& OR & $95 \%$ Cl & P value \\
Age & 1.022 & $0.991-1.055$ & 0.1682 \\
Hypertension & 0.857 & $0.484-1.518$ & 0.5965 \\
Dyslipidemia & 1.417 & $0.667-3.010$ & 0.4481 \\
Diabetes mellitus & 1.649 & $0.914-2.973$ & 0.0964 \\
Current smoking & 1.893 & $1.063-3.369$ & 0.0302 \\
\hline
\end{tabular}

Other abbreviations as in Table 2.

\begin{tabular}{|c|c|c|c|}
\hline & OR & $95 \% \mathrm{Cl}$ & $P$ value \\
\hline Age & 0.982 & $0.901-1.071$ & 0.6870 \\
\hline Hypertension & 2.201 & $0.451-10.731$ & 0.3292 \\
\hline Dyslipidemia & 0.889 & $0.182-4.351$ & 0.8849 \\
\hline Diabetes mellitus & 9.234 & $2.902-29.382$ & 0.0002 \\
\hline Current smoking & 2.383 & $0.566-10.023$ & 0.2362 \\
\hline
\end{tabular}

Other abbreviations as in Table 2.

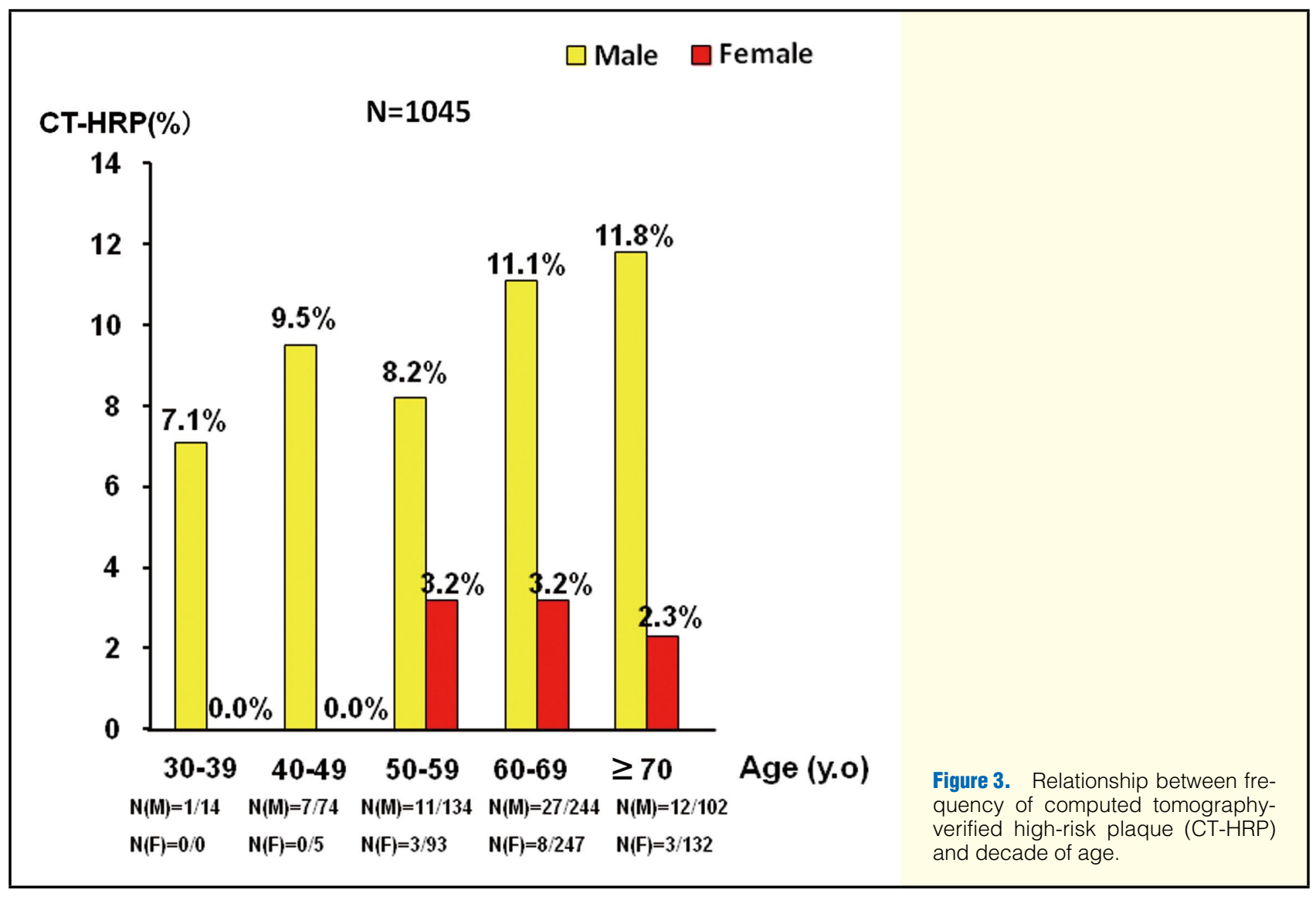

was more frequent in men than in women in all age groups. No CT-HRP was observed in women in their $40 \mathrm{~s}$ or younger (Figure 3).

\section{Discussion}

It has been reported that, in general, approximately $60 \%$ of rupture-prone vulnerable plaque is not associated with significant stenotic lesions, ${ }^{1,18}$ which makes it difficult to predict from clinical symptoms alone. Although current guidelines do not recommend coronary CTA for screening low-risk, asymptomatic patients for coronary heart disease,${ }^{19}$ and the clinical use of coronary CTA for the purpose of risk stratification of asymptomatic patients has not been established, ${ }^{20}$ recent technological advances in coronary CTA make it possible to reduce the dose of contrast medium and radiation exposure, suggesting that the indication for coronary CTA is broader than before. The present study regarding predictors identifying CT-
HRP, which has been reported to lead to ACS within 1 year with a probability of $11 \%,{ }^{6}$ is expected to provide useful information on higher-risk stratification, especially of intermediateand high-risk patients presenting with no or atypical symptoms based on FRS.

The Agatston score is closely associated with the presence of coronary atherosclerosis and it is well established that the greater the Agatston score, the higher the likelihood of cardiac events. ${ }^{21,22}$ The present study demonstrated that the prevalence of CT-HRP with an Agatston score >500 and >250 was lower in the intermediate-risk and high-risk groups, respectively. It has been reported that culprit lesions show acute coronary thrombus in approximately $50-60 \%$ of cases of coronary sudden death, and the remainder includes severe ischemic heart disease or arrhythmia. ${ }^{23}$ In other words, cardiac events are not necessarily caused by CT-HRP. Although it is thought that cardiac death because of severe cardiac ischemia and arrhythmia occurs if coronary atherosclerosis progresses, it is also 
thought that vulnerable plaque develops if the Agatston score, which indicates the accumulation of atherosclerosis, is high, because the progression of atherosclerotic change is not homogeneous in the 3 major coronary arteries. Autopsy study has suggested large variation in the degree of calcification within coronary atherosclerotic lesions in patients with sudden coronary death. ${ }^{24}$ On this point, the present results are consistent with those of previous reports with regard to Agatston scores. ${ }^{21,22}$ It is said that, in general, atherosclerosis develops stepwise in stages. ${ }^{3,25,26}$ Rupture-prone vulnerable plaque with a large necrotic core and thin fibrous cap containing many inflammatory cells such as macrophages, and characterized by positive remodeling, ${ }^{4,27}$ develops at a relatively early stage. In particular, positive remodeling refers to compensatory enlargement of the vessel diameter to maintain the lumen during the early stage of plaque progression. ${ }^{28,29}$ Because extensive calcification appears at the late stage of atherosclerotic progression, the prevalence of CT-HRP characterized by positive remodeling, low-density plaque and none or spotty calcification could decrease in extensive calcified lesions such as those with Agaston score $>500$. The low prevalence of CT-HRP in patients with Agatston score $>500$ was considered to be a reasonable result. In contrast, the prevalence of CT-HRP in Agatston $=0$ patients was $3.3 \%$ in the intermediate-risk group and $7.0 \%$ in the high-risk group, respectively, which implies that the presence of CT-HRP cannot be excluded in patients with Agatston score $=0$.

On logistic regression analysis, the significant predictors for CT-HRP were male sex, DM and current smoking in intermediate- and high-risk subjects. In particular, multivariate analysis identified male sex as the most powerful predictor. It has been reported that the age-related risk for coronary artery disease is lower in women than in men, and the risk of death from coronary artery disease in women is similar to that in men 10 years younger. ${ }^{30}$ Furthermore, the younger a patient's age, the greater the ratio of postmyocardial infarction mortality in women to that in men. ${ }^{31}$ In the present study, the frequency of CT-HRP was higher in men than in women in each generation, but did not increase with age. This does not support the report stating that the risk of death from coronary artery disease in women is similar to that in men 10 years younger. In addition, CT-HRP was observed in approximately $10 \%$ of the male patients in all age groups, but in none of the female patients younger than 50 years old. In contrast, DL was not a significant predictor for CT-HRP. Although multivariate analysis for various lipid profiles was attempted, total cholesterol and LDL-C were not significant predictors. The development of medical therapy for plaque stability, such as statins, might be relevant to this result. In fact, $78.8 \%$ of all study subjects were defined as having DL and $60 \%$ of patients with DL were taking statins. The mean LDL-C level was $126.2 \mathrm{mg} / \mathrm{dl}$, which is low. On the other hand, univariate analysis using logistic analysis revealed that TG and HDL-C were significant predictors for CT-HRP. Although only DL was used as a predictor in the multivariate analysis in the present study, further study of the lipid profile as a predictor for CT-HRP is needed. In analysis according to male and female sex, logistic regression analysis revealed significant predictors for CT-HRP to be only current smoking in males and DM in females. These results suggest that, when determining the need for coronary CTA for coronary risk stratification, it is necessary to consider the patient's age and sex, as well as the coronary risk factors.

We demonstrated the usefulness of coronary CTA for risk re-stratification of asymptomatic patients or those with atypical chest symptoms. CT-HRP is regarded as rupture-prone vul- nerable plaque, and characterized by positive remodeling and low attenuation, but, to date, only Motoyama et al have reported the frequency with which CT-HRP actually result in ACS and patients with known coronary artery disease were also included in thier study. ${ }^{6}$ In the present study, we did not observe how the frequency of CT-HRP results in ACS. Further, larger clinical trials are needed. Because CT values are influenced by factors such as the concentration of contrast medium in the coronary artery, degree of stenosis of coronary lesions, and tube voltage, ${ }^{32}$ the cut-off levels should be further evaluated.

\section{Study Limitations}

Subjects were patients who underwent coronary CTA despite being either asymptomatic or presenting with atypical chest symptoms. The subject group could be biased because the indication for coronary CTA was determined only by the outpatient physician. It is considered, however, that subjects were similar to a real-world population in clinical practice.

The FRS has been used in an American community and may not necessarily apply to Japanese populations. Additionally, the FRS is not well regarded as a predictor of coronary plaque vulnerability, but rather as a predictor of 10 year "hard" cardiovascular events. The combination of FRS and CT-HRP considered as rupture-prone vulnerable plaques, which account for approximately $60 \%$ of coronary lesion with thrombi, could be useful for cardiac event risk re-stratification, because it is considered that the vast majority of sudden coronary deaths are either the immediate result or a sequel of acute coronary arterial thrombosis. ${ }^{33}$

\section{Conclusions}

Among asymptomatic subjects and those presenting with atypical chest pain in the more than intermediate-risk group, coronary CTA results are contributory to the FRS. Although the prevalence of CT-HRP is decreased for Agatston score $>500$ in intermediate-risk subjects, it decreases for Agatston score $>250$ in the high-risk group. Male sex, DM, and current smoking are independent predictors of vulnerable plaque in subjects with more than an intermediate risk.

\section{References}

1. Falk E, Shah PK, Fuster V. Coronary plaque disruption. Circulation 1995; 92: 657-671.

2. Braunwald E. Epilogue: What do clinicians expect from imagers? $J$ Am Coll Cardiol 2006; 47(Suppl): C101-C103.

3. Naghavi M, Libby P, Falk E, Casscells SW, Litovsky S, Rumberger J, et al. From vulnerable plaque to vulnerable patient: A call for new definitions and risk assessment strategies; Part I. Circulation 2003; 108: $1664-1672$.

4. Narula J, Finn AV, Demaria AN. Picking plaques that pop. J Am Coll Cardiol 2005; 45: 1970-1973.

5. Motoyama S, Kondo T, Sarai M, Sugiura A, Harigaya H, Sato T, et al. Multislice computed tomographic characteristics of coronary lesions in acute coronary syndromes. J Am Coll Cardiol 2007; 50: 319326.

6. Motoyama S, Sarai M, Harigaya H, Anno H, Inoue K, Hara T, et al. Computed tomographic angiography characteristics of atherosclerotic plaques subsequently resulting in acute coronary syndrome. $J \mathrm{Am}$ Coll Cardiol 2009; 54: 49-57.

7. Achenbach S, Marwan M, Ropers D, Schepis T, Pflederer T, Anders K, et al. Coronary computed tomography angiography with a consistent dose below $1 \mathrm{mSv}$ using prospectively electrocardiogram-triggered high-pitch spiral acquisition. Eur Heart J 2010; 31: 340-346.

8. Alkaghi H, Stolzmann P, Desbiolles L, Baumueller S, Goetti R, Plass $\mathrm{A}$, et al. Low-dose, 128-slice, dual-source CT coronary angiography: Accuracy and radiation dose of the high-pitch and the step-and-shoot mode. Heart 2010; 96: 933-938. 
9. Hausleiter J, Martinoff S, Hadamitzky M, Martuscelli E, Pschierer I, Feuchtner GM, et al. Image quality and radiation dose exposure with a low tube voltage protocol for coronary CT angiography. J Am Coll Cardiol Imaging 2010; 3: 1113-1123.

10. Zhang C, Zhang Z, Yan Z, Xu L, Yu W, Wang R. 320-row CT coronary angiography: Effect of 100-kV tube voltage on image quality, contrast volume, and radiation dose. Int J Cardiovasc Imaging 2011; 27: $1059-1068$.

11. LaBounty TM, Leipsic J, Poulter R, Wood D, Johnson M, Srichai $\mathrm{MB}$, et al. Coronary CT angiography of patients with a normal body mass index using $80 \mathrm{kVp}$ versus $100 \mathrm{kVp}$ : A prospective, multicenter, multivendor randomized trial. Am J Roentgenol 2011; 197: W860W867.

12. Wilson PW, D'Agostino RB, Levy D, Belanger AM, Silbershatz H, Kannel WB. Prediction of coronary heart disease using risk categories. Circulation 1998; 97: 1837-1847.

13. Pryor DB, Harrell FE Jr, Lee KL, Califf RM, Rosati RA. Estimating the likelihood of significant coronary artery disease. Am J Med 1983; 75: $771-780$.

14. Pryor DB, Shaw L, McCants CB, Lee KL, Mark DB, Harrell FE Jr, et al. Value of the history and physical in identifying patients at increased risk for coronary artery disease. Ann Intern Med 1993; 118: $81-90$.

15. Raff GL, Abidov A, Achenbach S, Berman DS, Boxt LM, Budoff MJ, et al; Society of Cardiovascular Computed Tomography. SCCT guidelines for the interpretation and reporting of coronary computed tomographic angiography. J Cardiovasc Comput Tomogr 2009; 3: 122 136.

16. Agatston AS, Janowitz WR, Hildner FJ, Zusmer NR, Viamonte M Jr, Detrano R. Quantification of coronary artery calcium using ultrafast computed tomography. J Am Coll Cardiol 1990; 15: 827-832.

17. Fujimoto S, Kondo T, Narula J. Evaluation of plaque morphology in coronary computed tomographic angiography. Cardiol Clin 2012; 30: $69-75$

18. Virmani R, Burke AP, Kolodgie FD, Farb A. Vulnerable plaque: The pathology of unstable coronary lesions. J Interv Cardiol 2002; 15: 439-446

19. Hendel RC, Patel MR, Kramer CM, Poon M, Hendel RC, Carr JC, et al; American College of Cardiology Foundation Quality Strategic Directions Committee Appropriateness Criteria Working Group; American College of Radiology; Society of Cardiovascular Computed Tomography; Society for Cardiovascular Magnetic Resonance; American Society of Nuclear Cardiology; North American Society for Cardiac Imaging; Society for Cardiovascular Angiography and Interventions; Society of Interventional Radiology. ACCF/ACR/ SCCT/SCMR/ASNC/NASCI/SCAI/SIR 2006 appropriateness criteria for cardiac computed tomography and cardiac magnetic resonance imaging: A report of the American College of Cardiology Foundation Quality Strategic Directions Committee Appropriateness Criteria Working Group, American College of Radiology, Society of Cardiovascular Computed Tomography, Society for Cardiovascular Magnetic Resonance, American Society of Nuclear Cardiology, North American Society for Cardiac Imaging, Society for Cardiovascular Angiography and Interventions, and Society of Interventional Radiology. J Am Coll Cardiol 2006; 48: 1475-1497.
20. Chang HJ, Chung N. Clinical perspective of coronary computed tomographic angiography in diagnosis of coronary artery disease. Circ J 2011; 75: 246-252.

21. Greenland P, Bonow RO, Brundage BH, Budoff MJ, Eisenberg MJ, Grundy SM, et al. ACCF/AHA 2007 clinical expert consensus document on coronary artery calcium scoring by computed tomography in global cardiovascular risk assessment and in evaluation of patients with chest pain: A report of the American College of Cardiology Foundation Clinical Expert Consensus Task Force (ACCF/AHA Writing Committee to Update the 2000 Expert Consensus Document on Electron Beam Computed Tomography). J Am Coll Cardiol 2007; 49: $378-402$.

22. Yamamoto H, Ohashi N, Ishibashi K, Utsunomiya H, Kunita E, Oka $\mathrm{T}$, et al. Coronary calcium score as a predictor for coronary artery disease and cardiac events in Japanese high-risk patients. Circ J 2011; 75: $2424-2431$.

23. Farb A, Tang AL, Burke AP, Sessums L, Liang Y, Virmani R. Sudden coronary death: Frequency of active coronary lesions, inactive coronary lesions, and myocardial infarction. Circulation 1995; 92: $1701-1709$.

24. Burke AP, Weber DK, Kolodgie FD, Farb A, Taylor AJ, Virmani R. Pathophysiology of calcium deposition in coronary arteries. Herz. 2001; 26: 239-244.

25. Stary HC, Chandler AB, Dinsmore RE, Fuster V, Glagov S, Insull W Jr, et al. A definition of advanced types of atherosclerotic lesions and a histological classification of atherosclerosis: A report from the Committee on Vascular Lesions of the Council on Atherosclerosis, American Heart Association. Arterioscler Thromb Vasc Biol 1995; 15: $1512-1531$.

26. Fuster V, Moreno PR, Fayad ZA, Corti R, Badimon JJ. Atherothrombosis and high-risk plaque. Part I: Evolving concepts. J Am Coll Cardiol 2005; 46: 937-954.

27. Virmani R, Burke AP, Farb A, Kolodgie FD. Pathology of the vulnerable plaque. J Am Coll Cardiol 2006; 47: C13-C18.

28. Glagov S, Weisenberg E, Zarins CK, Stankunavicius R, Kolettis GJ. Compensatory enlargement of human atherosclerotic coronary arteries. N Engl J Med 1987; 316: 1371-1375.

29. Clarkson TB, Prichard RW, Morgan TM, Petrick GS, Klein KP. Remodeling of coronary arteries in human and nonhuman primates. JAMA 1994; 271: 289-294.

30. Lerner DJ, Kannel WB. Patterns of coronary heart disease morbidity and mortality in the sexes: A 26-year follow-up of the Framingham population. Am Heart J 1986; 111: 383-390.

31. Vaccarino V, Parsons L, Every NR, Barron HV, Krumholz HM, for the National Registry of Myocardial Infarction 2 Participants. Sexbased differences in early mortality after myocardial infarction. $N$ Engl J Med 1999; 341: 217-225.

32. Cademartiri F, Mollet NR, Runza G, Bruining N, Hamers R, Somers $\mathrm{P}$, et al. Influence of intracoronary attenuation on coronary plaque measurements using multislice computed tomography: Observations in an ex vivo model of coronary computed tomography angiography. Eur Radiol 2005; 15: 1426-1431.

33. Arbab-Zadeh A, Nakano M, Virmani R, Fuster V. Acute coronary events. Circulation 2012; 125: 1147-1156. 